\title{
Genomic analysis of pediatric cataract in Saudi Arabia reveals novel candidate disease genes
}

\author{
Mohammed A Aldahmesh, PhD ${ }^{1}$, Arif O Khan, MD ${ }^{1,2}$, Jawahir Y Mohamed, BSc ${ }^{1}$, Hadia Hijazi, BSc ${ }^{1}$, \\ Mohammed Al-Owain, MD,4, Abdulrahman Alswaid, MD ${ }^{5}$ and Fowzan S Alkuraya, MD 1,3,6
}

\begin{abstract}
Background: Pediatric cataract is an important preventable blinding disease. Previous studies have estimated $10-25 \%$ of cases to be genetic in etiology.
\end{abstract}

Methods: In an effort to characterize the genetics of cataract in our population, we have conducted a comprehensive clinical and genomic analysis (including autozygome and exome analysis) on a series of 38 index patients.

Results: Pediatric cataract is genetic in at least $79 \%$ of the study families. Although crystallins accounted for most of the mutant alleles, mutations in other genes were encountered, including recessive mutations in genes that usually cause the disease in a dominant manner. In addition, several novel candidate genes (MFSD6L, AKR1E2, RNLS, and CYP51A1) were identified.

Conclusion: Pediatric cataract is typically a genetic disease, usually autosomal recessive, in Saudi Arabia. Although defining a specific cataract phenotype can sometimes predict the genetic cause, genomic analysis is often required to unravel the causative mutation given the marked genetic heterogeneity. The identified novel candidate genes require independent confirmation in future studies.

Genet Med 2012:14(12):955-962

Key Words: autozygosity; crystallin; EPHA2; exome; FYCO1; GCNT2
The human crystalline lens is a key functional component in the visual axis that allows images of objects at a wide range of distances to be focused on the retinal photoreceptors for proper visual perception to take place. ${ }^{1}$ Embryologically, the lens also plays a critical role in the development of the entire eye through participating in the cross-talk between the lens placode and the optic vesicle. ${ }^{2}$ Transparency of the lens is achieved by virtue of a number of unique properties that are both structural and functional. ${ }^{3}$ For instance, lens fiber cells lack nuclei and organelles and are orchestrated in a highly structured pattern that minimizes intercellular spaces and endows a high refractive index. In addition, the lens is very rich in proteins, mostly crystallins, with unique optical properties.

Significant lenticular opacities, known as cataracts, cause significant morbidity especially in children because of their susceptibility to irreversible visual loss from amblyopia. Although the incidence of pediatric cataract is estimated at only 1-6 cases per 10,000 live births, in terms of lost functional years pediatric cataract causes a burden that rivals that of the much more common senile cataract. ${ }^{4,5}$ The complexity of lenticular development and its structure makes the lens particularly vulnerable to a range of insults that are both genetic and environmental in nature. Determining the etiology of cataract is particularly challenging in sporadic cases that lack family history, but familial occurrence of bilateral cataract is highly suggestive of a genetic etiology. This has to be taken into account when one examines available data on the breakdown of pediatric cataract etiology that suggest $10-25 \%$ to be genetic in etiology whereas $60 \%$ are idiopathic. $^{6}$

Within the "genetic" category of pediatric cataract, mutations in crystallins are the most common, accounting for nearly $50 \%$ of cases. ${ }^{7}$ However, more than 30 loci have been implicated. The disease genes for more than 20 of these have been identified, representing a wide range of cellular processes. The majority of these genes and loci (approximately two-thirds) are autosomal dominant, although some of these dominant genes can also cause cataract recessively. ${ }^{7}$ Although deducing the hereditary nature of multiplex cases may be straightforward, sporadic cases pose a significant challenge because they are labeled as "idiopathic" unless one performs comprehensive genetic analysis that takes into account the possibilities of de novo autosomal dominant, autosomal recessive, or X-linked inheritance. Given the significant genetic heterogeneity of pediatric cataract, such comprehensive analysis has been very difficult to implement.

To address this important gap in our knowledge of genetic causes of cataract, we have adopted a more comprehensive approach than what has previously been published. In this case series, we have devised and followed an algorithm that aims at maximizing the likelihood of identifying an underlying genetic etiology by taking advantage of such genomic tools as autozygome analysis and exome sequencing. In our population, we show that autosomal recessive inheritance accounts for the majority of familial cases. We also show that

${ }^{1}$ Department of Genetics, King Faisal Specialist Hospital and Research Center, Riyadh, Saudi Arabia; ${ }^{2}$ Division of Pediatric Ophthalmology, King Khaled Eye Specialist Hospital, Riyadh, Saudi Arabia; ${ }^{3}$ Department of Anatomy and Cell Biology, College of Medicine, Alfaisal University, Riyadh, Saudi Arabia; ${ }^{4}$ Department of Medical Genetics, King Faisal Specialist Hospital and Research Center, Riyadh, Saudi Arabia; ${ }^{5}$ Department of Pediatrics, King Abdulaziz Medical City, Riyadh, Saudi Arabia; ${ }^{6}$ Department of Pediatrics, King Khalid University Hospital and College of Medicine, King Saud University, Riyadh, Saudi Arabia. Correspondence: Fowzan S Alkuraya (falkuraya@kfshrc.edu.sa) 
the combined use of genomic tools can unravel an underlying genetic etiology in the majority of familial cases, including novel candidate disease genes that we report for the first time.

\section{MATERIALS AND METHODS Human subjects}

This is a case series of children referred to one of the authors from all regions of Saudi Arabia for the management of pediatric cataract (2004-2011). Patients were then referred for molecular diagnostic candidate gene testing based on the cataract phenotype, when applicable; otherwise, and if negative, they signed a written consent to participate in a research protocol. A member of the Developmental Genetics Unit of the King Faisal Hospital and Research Center provided genetic testing and counseling following full informed consent. Venous blood samples were collected from patients, their affected and unaffected siblings (when applicable) and parents. Detailed ophthalmological examination, including slit lamp examination, was performed on all cases and their parents when available. Sporadic cases were defined as those with negative family history whereas those with positive family history were defined as familial cases. Patients with suspected or known nongenetic etiology (e.g., infection, trauma) were excluded, as were patients with known syndromes associated with pediatric cataract (e.g., Down syndrome, Stickler syndrome). The study was approved by the King Faisal Specialist Hospital and Research Center institutional review board (RAC no. 2070023).

\section{Molecular analysis}

Genotyping and autozygosity scan. DNA was extracted from whole blood using a standard protocol followed by genome-wide genotyping using the Axiom SNP Chip platform (Affymetrix, Santa Clara, CA) following the manufacturer's protocol. Autozygosity scan was performed using autoSNPa. ${ }^{8}$

Exome sequencing and analysis. Exome capture was performed using TruSeq Exome Enrichment kit (Illumina, San Diego, CA)following the manufacturer's protocol. Samples were prepared as an Illumina sequencing library, and in the second step, the sequencing libraries were enriched for the desired target using the Illumina Exome Enrichment protocol. The captured libraries were sequenced using an Illumina HiSeq 2000 Sequencer. The reads were mapped against UCSC hg19 (http://genome.ucsc.edu/) by BWA (http://bio-bwa.sourceforge.net/). The single-nucleotide polymorphisms and indels were detected by SAMTOOLS (http://samtools.sourceforge.net/).

Sequence analysis. Primers for candidate genes were designed using Primer3 for PCR with added M13 tails that were used for capillary sequencing. Primers were designed to cover all coding exons and at least $100 \mathrm{bp}$ of the flanking introns.

\section{RESULTS}

\section{Human subjects}

In total, 38 index patients were recruited representing 38 apparently unrelated families. Familial cases accounted for the majority of cases (63\%) and of these, autosomal recessive inheritance was the likely mode of inheritance in the majority (71\%). These two statistics are in stark contrast to what has been published earlier in the cataract literature and are likely accounted for by the unique population structure of Saudi Arabia with its high rate of consanguinity and large family size. A summary of the cataract phenotype and age of onset for each of the 38 index patients is shown in Supplementary Table S1 online. The algorithm followed in the analysis of these cases is outlined by the flowchart in Figure 1.

\section{Autozygome-guided mutation analysis}

For the 17 familial cases that were likely to have an autosomal recessive form of cataract, we hypothesized that the causative mutation would reside within a block of autozygosity given the high rate of consanguinity. Indeed, autozygosity scan identified a likely causative mutation in 11 cases $(65 \%)$ (Table 1). Seven of these 11 families harbored a previously reported autosomal recessive founder truncating mutation in CRYBB1 (c.171delG; p.Asn58ThrfsX107). ${ }^{9}$ The remaining four families harbored homozygous mutations in other known cataract genes, namely BFSP2, GCNT2, EPHA2, and FYCO1. Recessive mutations (seven in total) in GCNT2, which encodes glucosaminyl (N-acetyl) transferase 2, have been reported in Japanese, Taiwanese, Arab, and Pakistani families with congenital cataract and adult I blood group and encompass missense, nonsense, and genomic alterations. ${ }^{10-13}$ The missense mutation we report in this study (c.1040A>G; p.Tyr347Cys), which fully segregated with the phenotype in the family, is predicted to be pathogenic by in silico analysis and is very well conserved down to acorn worm and Xenopus. In addition, it is absent in 192 Saudi controls by Sanger sequencing and 160 Saudi controls by exome sequencing. Although EPHA2 mutations were originally identified in autosomal dominant cataract, a homozygous recessive mutation was subsequently identified in a Pakistani family. ${ }^{14-16}$ The mutation we report (c.1405T>C; p.Tyr469His), which affects a tyrosine residue in the fibronectin-III domain that is absolutely conserved across all known Ephrin type A receptor genes, is the second reported recessive mutation in this gene (Supplementary Figure $\mathbf{S 3}$ online). This is similar to our experience with the BFSP2 mutation, which we have previously reported as the first recessive mutation in this gene. ${ }^{17}$ FYCO1, encoding FYVE and coiled-coil domain containing 1, was only very recently reported to cause autosomal recessive cataract in 12 Pakistani families and one Arab family. ${ }^{18}$ Our study, therefore, represents the second report of FYCO1 mutation in congenital cataract.

In the remaining six families, no candidate gene was highlighted by the autozygosity scan or the candidate genes were excluded by direct sequencing; therefore, we proceeded with exome sequencing as described below. We also implemented 


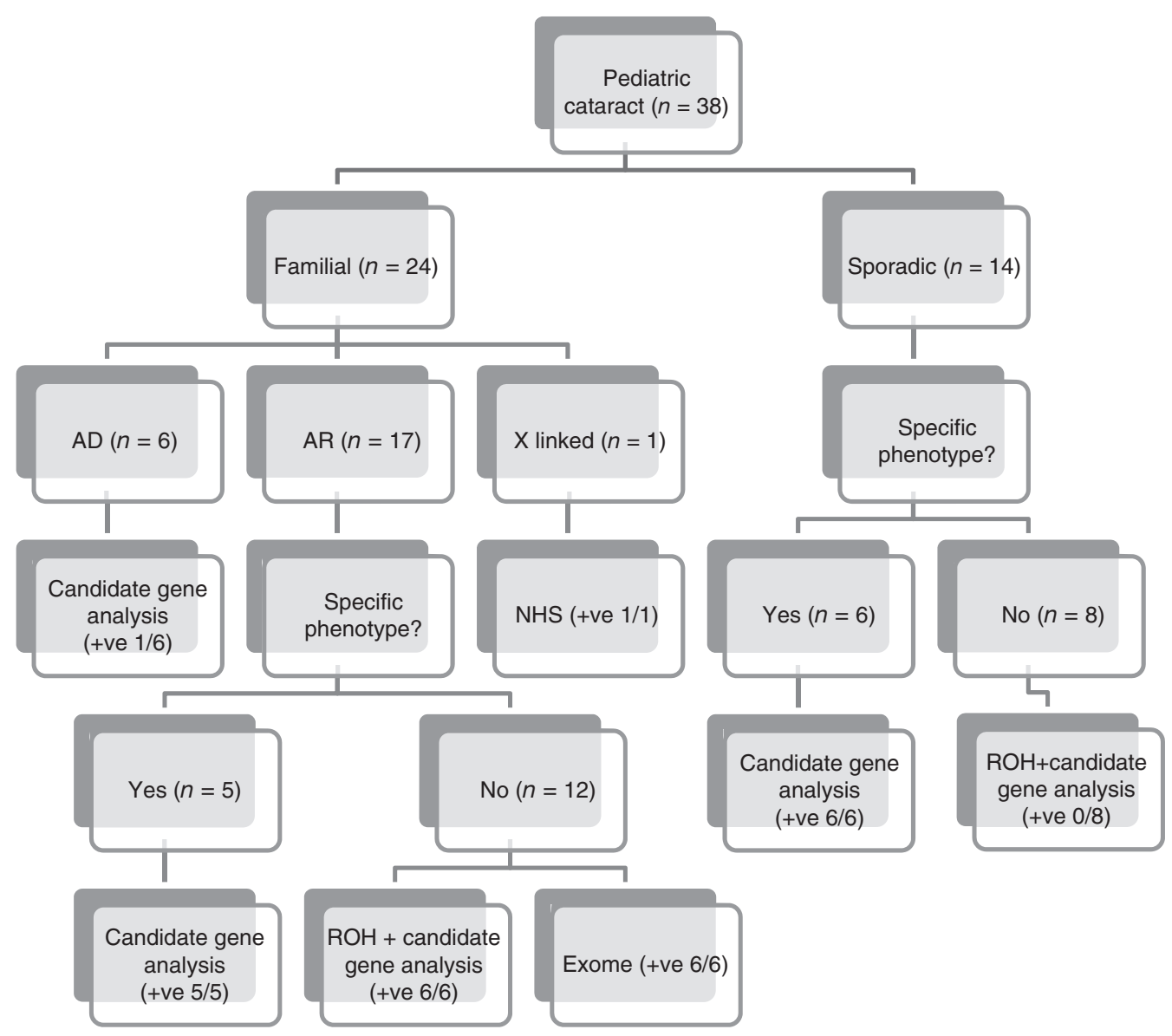

Figure 1 Workflow of the current study. AD, autosomal dominant; AR, autosomal recessive; NHS, Nance-Horan syndrome; ROH, runs of homozygosity; +ve, mutation-positive cases.

autozygosity scan in the eight sporadic cases of cataract in which candidate gene analysis did not reveal a pathogenic mutation. In each of these cases, we included all known human and mouse cataract genes regardless of the mode of inheritance (Table 1), but we failed to identify any pathogenic mutation.

\section{Exome sequencing}

We have achieved on average 50X on-target coverage. The filtration strategy and resulting variants are summarized in Figure 2 and Table 2. In Family Cata_DGU-13, a splice-site variant in CYP27A1 (c.1263+1G>A) was identified and confirmed to abolish the consensus donor site and lead to an aberrant transcript. Mutations in this gene are known to cause cerebrotendinous xanthomatosis (OMIM no. 213700), a rare progressive multisystem lipid-storage disorder with age-dependent penetrance for which juvenile cataract could the presenting sign. ${ }^{19}$ Indeed, at the time of their presentation ( $\sim 7$ years of age), these two siblings had normal systemic examination and no neurological signs of this disease, which usually manifests around puberty. When specifically questioned, the parents confirmed that both affected siblings had suffered from uncontrolled early childhood diarrhea, a known manifestation of the condition.

In addition to revealing the above known cause of cataract, exome sequencing revealed four novel candidates and one recently published. In Family Cata_DGU-14, we were left, after applying all the filters shown in Figure 2, with a novel variant in MFSD6L (c.T1616C:p.L539P) that segregated with the phenotype of cataract and severe psychomotor delay in the family. This variant is predicted to be highly pathogenic based on conservation level and protein structure. Similarly, the cataract phenotype in Family Cata_DGU-16 was found to segregate with a novel missense variant in CYP51A1, encoding a member of the CYP450 family of enzymes also known as lanosterol 14- $\alpha$ demethylase, which is predicted to be pathogenic at the conservation and structural level. These two novel variants were absent in 192 Saudi controls by Sanger sequencing and 160 Saudi controls by exome sequencing.

In contrast to these two missense variants, exome sequencing and subsequent filtration highlighted three truncating variants in the remaining three families. In Family Cata_DGU-12, a splice-site variant in $A G K$, encoding acylglycerol kinase, was identified and published elsewhere as the first reported recessive mutation in this lipid metabolizing enzyme in nonsyndromic cataract. ${ }^{20}$ In Family Cata_DGU-15, a splice-site variant (c.582+1G $>A$ ) in AKR1E2, encoding aldo-ketoreductase family 1 member E2, segregated with the phenotype. Unfortunately, because this gene does not seem to be expressed in the blood, we were unable to confirm the effect on splicing even though 


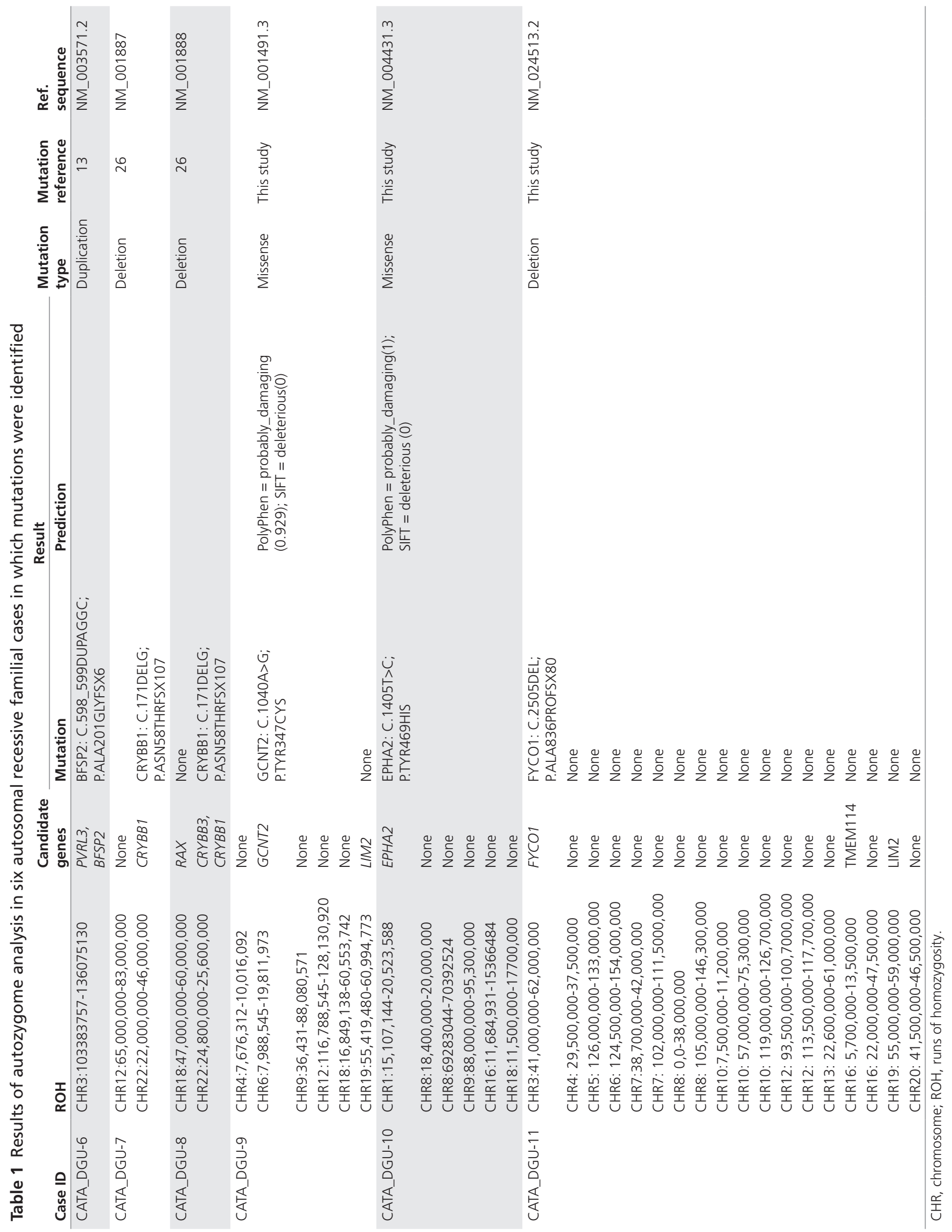




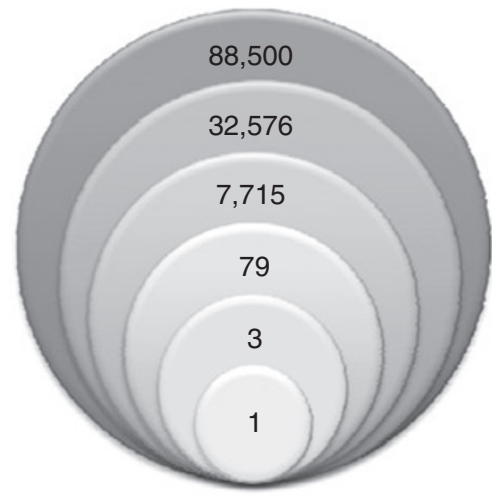

Cata_DGU-13

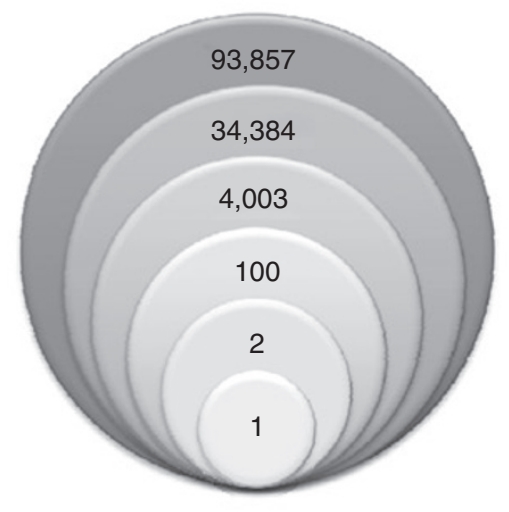

Cata_DGU-16

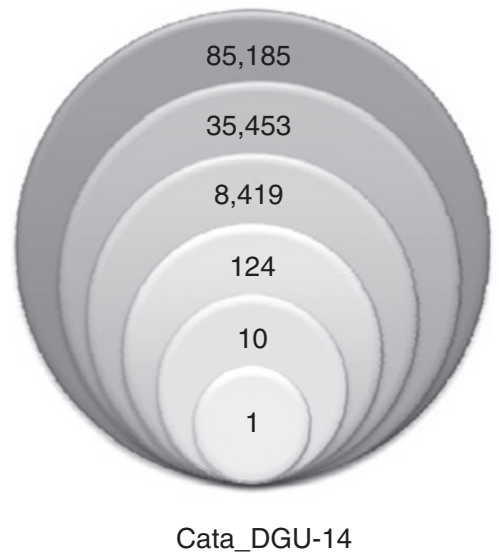

Total exome variants

Homozygous

Coding/splice

Absent in dbSNP

Within the autozygome

Absent in Saudi exomes
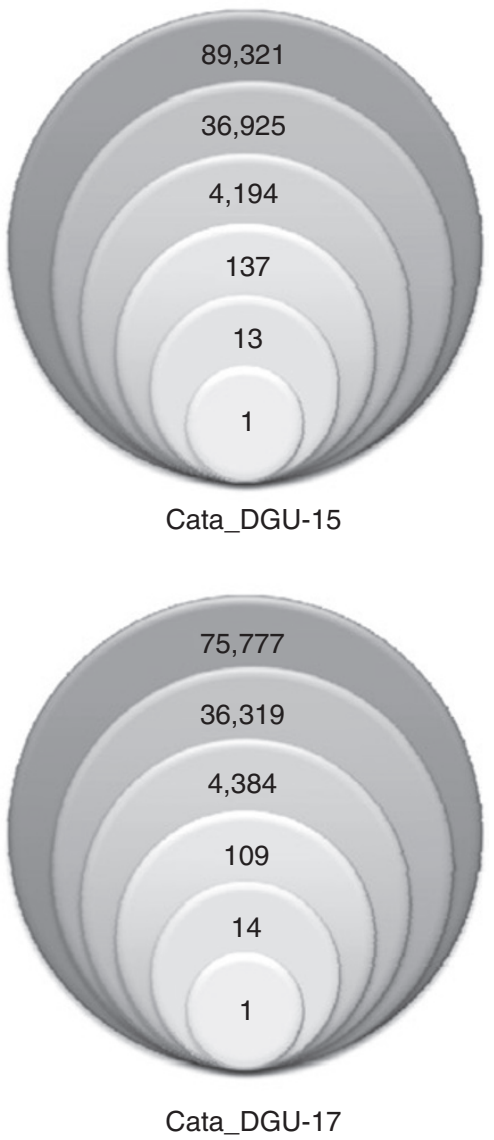

Figure 2 Filters used in analyzing the exome data.

it is predicted in silico to completely abolish the consensus donor site. In Family Cata_DGU-17, a truncating variant (c.215_216delinsT: p.Lys72Ilefs*10) was identified in RNLS, encoding renalase.

All novel mutations and candidate variants in this study are shown in Supplementary Figure S1 online and their segregation is shown in Supplementary Figure S4 online. The conserved nature of the novel missense variants is shown in Supplementary Figure S2 online.

\section{Candidate gene analysis}

Family Cata_DGU-25 was consistent with X-linked inheritance, and sequencing of NHS revealed the presence of a mutation, which we reported earlier. ${ }^{21}$ In sporadic cases, we sequenced the likely candidate when the cataract phenotype was known to be highly predictive of a specific gene. Indeed, the cataract phenotype was specific in 6 of the 14 sporadic cases. All five patients with pulvurent cataract were found to harbor a founder homozygous CRYBB1 mutation despite the fact that this phenotype is known to be genetically heterogeneous. ${ }^{22}$ Although cerulean cataract is also genetically heterogeneous, the only patient with cerulean cataract was sequenced first for CRYGD and found to harbor a dominant P23T mutation. ${ }^{23}$ In the remaining eight patients, we attempted autozygosity scan and candidate gene analysis in search of pathogenic mutations, but none were identified.

\section{DISCUSSION}

To our knowledge, this is the largest comprehensive genetic study of cataract that involves the combined use of such genomic tools as autozygome and exome sequencing. Although this is not a population-based study, the enrollment of all eligible cases referred to one of the authors from all parts of the country over a period of 5 years suggests that our results may reflect a population pattern.

Our study shows that at least $79 \%$ of pediatric cataract in our cohort is genetic in etiology. Although it is easy to infer the genetic nature in the 24 cases that are familial, we show that a significant percentage of sporadic cases (43\%) can also be traced to a single gene defect. These findings are remarkably different from previous estimates; we propose that the characteristically high consanguinity and large family size in the study population are important factors in shaping this difference. Although all cases of pediatric cataract were equally likely to be referred, we cannot rule out the hypothetical possibility that those with family history were more likely to be referred and/ or be more motivated to accept the referral. However, even if this factor inflated the percentage of familial cases, one cannot 


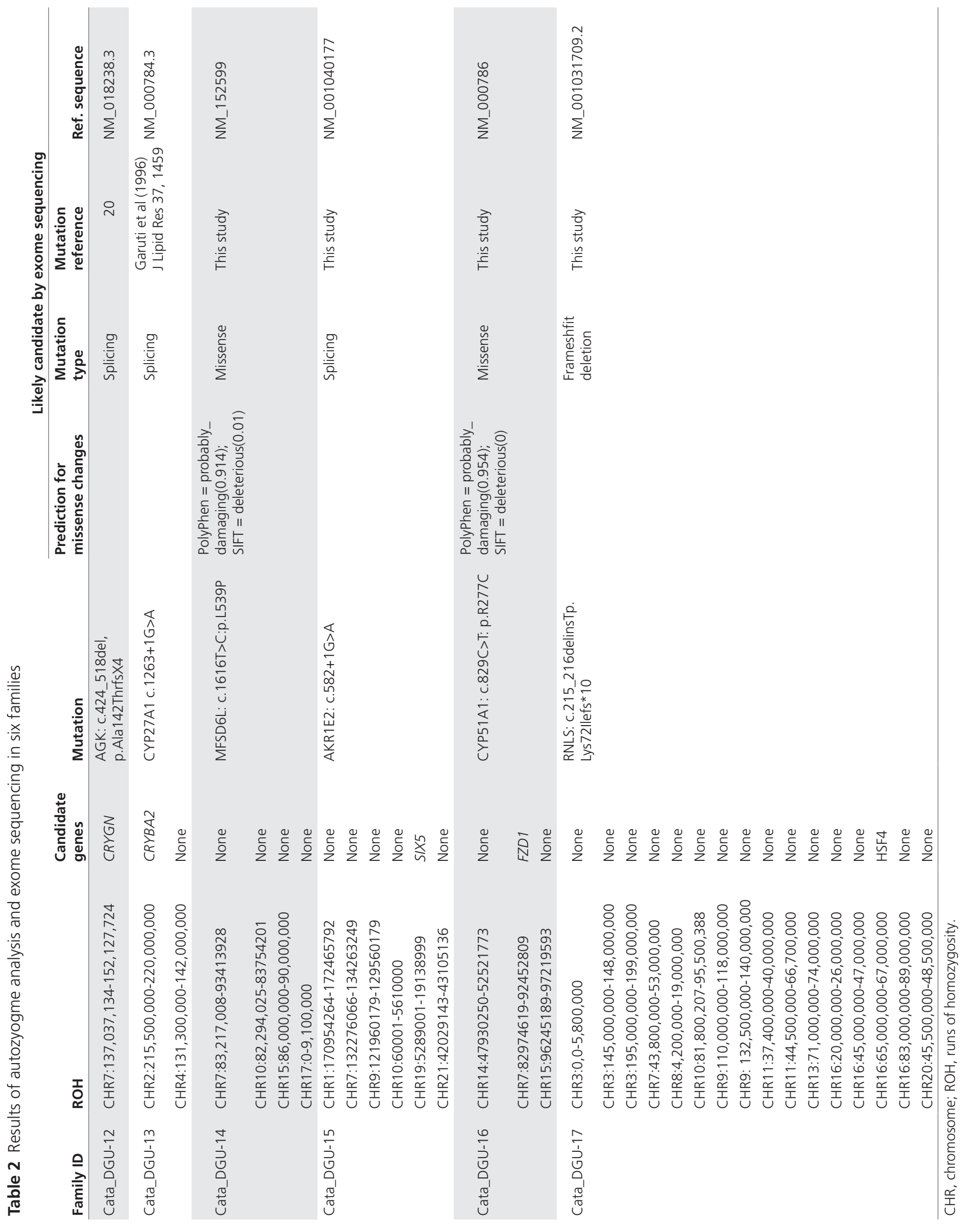


invoke this as an explanation of the high percentage of genetic causes among sporadic cataract cases.

The use of autozygome analysis to identify the underlying mutation in genetically heterogeneous disorders has been well documented. Indeed, our data show that this approach helped to uncover the underlying mutation in about $71 \%$ of familial autosomal recessive cases. Although 6 of the 14 sporadic cases did have an underlying autosomal recessive mutation that was identified by directly sequencing the likely candidate based on the specific cataract phenotype, it is important to note that autozygome analysis would have been able to highlight this gene otherwise. Thus, it appears that autozygome analysis in sporadic cases and in familial recessive cases is a reasonable first-tier approach in our population and perhaps other highly consanguineous populations.

Exome sequencing is an extremely powerful new tool in the setting of Mendelian diseases, particularly those that are autosomal recessive. We show that this approach revealed a likely candidate in all six familial cases that are suggestive of recessive inheritance in which autozygome analysis failed to highlight a known cataract gene. It is worth highlighting that the family with cerebrotendinous xanthomatosis could have been identified by autozygome analysis, but we were unaware that juvenile cataract could be the major presenting sign and hence CYP27A1 was not pursued with sequencing even though it was in fact flagged initially by autozygome analysis. A recent report does highlight that cataract (and infantile diarrhea) could indeed be the first presenting sign of the disease. ${ }^{19}$ None of the four novel candidate genes has passed the gold standard of causality by demonstrating that several patients with cataract have different mutations in this gene as has been shown for $A G K \cdot{ }^{20,24,25}$ An argument for and against the candidacy of these four genes is presented in this study although we acknowledge that only through the identification of independent mutations among patients with the same phenotype can one unequivocally designate them as cataract disease genes.

AKR1E2 has been previously shown to be among the top two percentiles of lens-enriched genes in the developing murine lens. ${ }^{26}$ Although the splicing mutation is predicted to completely abolish the consensus donor site, we were unable to confirm this experimentally due to apparent lack of expression in blood. This enzyme catalyzes the nicotinamide adenine dinucleotide phosphate-oxidase-dependent reduction of glycogen-derived 1,5-anhydro-D-fructose (AF) to 1,5-anhydro-D-glucitol (AG). Aldo-ketoreductases have attracted interest because of their presumed role in osmotic cataract in patients with diabetes in whom chronically elevated glucose is thought to be converted in excess into sorbitol, leading to cellular swelling and cataract formation. Thus, one tentative link between AKR1E2 and cataract is to hypothesize that AF accumulation as a result of AKR1E2 deficiency may have forced the conversion of AF to other polyols that generate higher osmotic pressure.

$R N L S$ encodes a distinct class of FAD-dependent monoamine oxidases known as renalase. Unlike the other well-studied FAD-dependent monoamine oxidases MAO-A and MAO-B, renalase is a secreted protein that catabolizes circulating catecholamines. ${ }^{27}$ Our mutation truncates the 342aa protein to 82aa only (10 amino acids of which are novel amino acids introduced by the frameshift) so it is likely to be an inactivating mutation. However, given that catecholamine metabolism is the only known function of this protein, it is unclear how it could be related to cataractogenesis. It may be tempting to hypothesize that this mutation represents a low-frequency variant that modifies the blood pressure phenotype in the general population. However, none of the $>10,000$ sequenced alleles listed in the Exome Variant Server is clearly inactivating. Thus, it remains possible that this is a true disease causing mutation in the setting of this family's cataract.

CYP51A1 is the only member of the CYP450 family of proteins that has a catalytically conserved function (sterol 14ademethylation) across biological kingdoms and is ubiquitously expressed in all mammalian tissues. ${ }^{28,29}$ Many disorders that involve sterol metabolism manifest with cataract in addition to a more generalized systemic involvement, e.g., cerebrotendinous xanthomatosis, lathosterolosis, Smith-Lemli-Opitz syndrome, and chondrodysplasia punctata 2 . Thus, it is possible that abnormal sterol metabolism in the context of CYP51A1 deficiency may also lead to cataract. Unfortunately, mice that completely lack this gene die at midgestation, so it is not possible to discern a potential lenticular phenotype. ${ }^{30}$

Perhaps the most challenging novel candidate is MFSD6L, which we identified in a family with cataract and severe psychomotor retardation. Very little is known about this gene other than that it encodes a multipass membrane protein that bears similarity to Major Facilitator Superfamily Domain-Containing Protein 6, which is known to be mutated in neuronal ceroid lipofuscinosis, a progressive disease that affects the brain and eye. The only findings that support a possible causal role for the variant we identified is its high pathogenicity score based on conservation and structure in addition to what we routinely did for all the above variants that remained after filtration, i.e., confirming their absence in 192 Saudi controls by direct sequencing (note that their absence in 160 Saudi exomes was among the filtration criteria) and their segregation with the phenotype within the family.

In conclusion, pediatric cataract appears to be mostly genetic in Saudi Arabia. Marked genetic heterogeneity is observed as is the predominance of autosomal recessive inheritance, which should be considered even in sporadic cases. Autozygomeguided mutation analysis has a high yield as a first-tier test, but the constantly dropping cost of exome sequencing suggests that the latter may soon be the first choice clinical test for children with cataract. We are currently investigating the utility of exome sequencing in sporadic and dominant cases of cataract. All families in which a gene was identified have been counseled regarding their reproductive choices. The very high interest in preventative genetic services makes a compelling case to pursue a potential genetic cause in all patients with pediatric cataract despite the challenges in the pursuit of identification of the cause in this etiologically heterogeneous disease. 


\section{SUPPLEMENTARY MATERIAL}

Supplementary material is linked to the online version of the paper at http://www.nature.com/gim

\section{ACKNOWLEDGMENTS}

We thank the patients and their families for their participation. We thank the Genotyping and Sequencing Core Facilities at King Faisal Specialist Hospital and Research Center for their technical help. This work was supported in part by a King Abdulaziz City for Science and Technology grant 08MED497-20 (to F.S.A.) and Dubai-Harvard Foundation for Medical Research Collaborative Research Grant (to F.S.A.).

\section{DISCLOSURE}

The authors declare no conflict of interest.

\section{REFERENCES}

1. Tardieu A. alpha-Crystallin quaternary structure and interactive properties control eye lens transparency. Int J Biol Macromol 1998;22:211-217.

2. Donner AL, Lachke SA, Maas RL. Lens induction in vertebrates: variations on a conserved theme of signaling events. Semin Cell Dev Biol 2006;17: 676-685.

3. Delaye M, Tardieu A. Short-range order of crystallin proteins accounts for eye lens transparency. Nature 1983;302:415-417.

4. Bermejo E, Martínez-Frías ML. Congenital eye malformations: clinicalepidemiological analysis of 1,124,654 consecutive births in Spain. Am J Med Genet 1998;75:497-504.

5. Gilbert C, Foster A. Childhood blindness in the context of VISION 2020--the right to sight. Bull World Health Organ 2001;79(3):227-232.

6. Hejtmancik JF. Congenital cataracts and their molecular genetics. Semin Cell Dev Bio/ 2008;19:134-149.

7. Huang $\mathrm{B}, \mathrm{He}$ W. Molecular characteristics of inherited congenital cataracts. Eur J Med Genet 2010;53:347-357.

8. Carr IM, Flintoff KJ, Taylor GR, Markham AF, Bonthron DT. Interactive visual analysis of SNP data for rapid autozygosity mapping in consanguineous families. Hum Mutat 2006;27:1041-1046.

9. Khan AO, Aldahmesh MA, Mohamed JY, Alkuraya FS. Clinical and molecular analysis of children with central pulverulent cataract from the Arabian Peninsula. Br J Ophthalmol 2012; 96:650-655.

10. Borck G, Kakar N, Hoch J, et al. An Alu repeat-mediated genomic GCNT2 deletion underlies congenital cataracts and adult i blood group. Hum Genet 2012;131:209-216.

11. Inaba N, Hiruma T, Togayachi A, et al. A novel I-branching beta-1,6-Nacetylglucosaminyltransferase involved in human blood group I antigen expression. Blood 2003;101:2870-2876.

12. Pras E, Raz J, Yahalom V, et al. A nonsense mutation in the glucosaminyl (N-acetyl) transferase 2 gene (GCNT2): association with autosomal recessive congenital cataracts. Invest Ophthalmol Vis Sci 2004;45:1940-1945.
13. Yu LC, Twu YC, Chou ML, et al. The molecular genetics of the human I locus and molecular background explain the partial association of the adult i phenotype with congenital cataracts. Blood 2003;101:2081-2088.

14. Kaul H, Riazuddin SA, Shahid M, et al. Autosomal recessive congenital cataract linked to EPHA2 in a consanguineous Pakistani family. Mol Vis 2010; 16:511-517.

15. Shiels A, Bennett TM, Knopf $\mathrm{HL}$, et al. The EPHA2 gene is associated with cataracts linked to chromosome 1p. Mol Vis 2008;14:2042-2055.

16. Zhang $T$, Hua $R$, Xiao W, et al. Mutations of the EPHA2 receptor tyrosine kinase gene cause autosomal dominant congenital cataract. Hum Mutat 2009:30:E603-E611.

17. Aldahmesh MA, Khan AO, Mohamed J, Alkuraya FS. Novel recessive BFSP2 and PITX3 mutations: insights into mutational mechanisms from consanguineous populations. Genet Med 2011;13:978-981.

18. Chen J, Ma Z, Jiao X, et al. Mutations in FYCO1 cause autosomal-recessive congenital cataracts. Am J Hum Genet 2011;88:827-838.

19. Monson DM, DeBarber AE, Bock CJ, et al. Cerebrotendinous xanthomatosis: a treatable disease with juvenile cataracts as a presenting sign. Arch Ophthalmol 2011;129:1087-1088.

20. Aldhamesh MA, Khan AO, Mohamed JY, Alghamdi MH, Alkuraya FS. Identification of a truncation mutation of the acylglycerol kinase (AGK) gene in a novel autosomal recessive cataract locus. Hum Mutat. 2012;33:960-962.

21. Khan AO, Aldahmesh MA, Mohamed JY, Alkuraya FS. Phenotypegenotype Correlation in Potential Female Carriers of X-linked Developmental Cataract (Nance-Horan Syndrome). Ophthalmic Genet 2012; 33:89-95

22. Khan AO, Aldahmesh MA, Mohamed JY, Alkuraya FS. Clinical and molecular analysis of children with central pulverulent cataract from the Arabian Peninsula. Br J Ophthalmol 2012;96:650-655.

23. Khan AO, Aldahmesh MA, Ghadhfan FE, Al-Mesfer S, Alkuraya FS. Founder heterozygous P23T CRYGD mutation associated with cerulean (and coralliform) cataract in 2 Saudi families. Mol Vis 2009;15:1407-1411.

24. Calvo SE, Compton AG, Hershman SG, et al. Molecular diagnosis of infantile mitochondrial disease with targeted next-generation sequencing. Sci Trans/ Med 2012:4(118):118ra110

25. Mayr JA, Haack TB, Graf E, et al. Lack of the mitochondrial protein acylglycerol kinase causes Sengers syndrome. Am J Hum Genet 2012; 90:314-320

26. Lachke SA, Ho JW, Kryukov GV, et al. iSyTE: integrated Systems Tool for Eye gene discovery. Investig Ophthalmo/ Vis Sci. 2012; 53:1617-1627.

27. Xu J, Li G, Wang P, et al. Renalase is a novel, soluble monoamine oxidase that regulates cardiac function and blood pressure. J Clin Invest 2005;115:12751280

28. Lepesheva GI, Virus C, Waterman MR. Conservation in the CYP51 family. Role of the $B^{\prime}$ helix/BC loop and helices $F$ and $G$ in enzymatic function. Biochemistry 2003:42:9091-9101.

29. Lepesheva GI, Waterman MR. Sterol 14alpha-demethylase cytochrome P450 (CYP51), a P450 in all biological kingdoms. Biochim Biophys Acta 2007; 1770:467-477.

30. Keber $\mathrm{R}$, Motaln $\mathrm{H}$, Wagner KD, et al. Mouse knockout of the cholesterogenic cytochrome P450 lanosterol 14alpha-demethylase (Cyp51) resembles AntleyBixler syndrome. J Biol Chem 2011;286:29086-29097. 